\title{
MSTN mutant promoted bovine muscle satellite cell proliferation by regulating the binding of SMAD2/SMAD3 with CDKN1C
}

\section{Li Gao}

Baotou Teachers College

\section{Miao-Miao Yang}

State Key Laboratory of Reproductive Regulation \& Breeding of Grassland Livestock, Inner Mongolia University

\section{Ming-Juan Gu}

State Key Laboratory of Reproductive Regulation \& Breeding of Grassland Livestock, Inner Mongolia University

\section{Yun-Peng Liu}

Baotou Teachers College

\section{Cai-Hong Bu}

Baotou Teachers College

\section{Peng Cheng}

\section{Baotou Teachers College}

Chunling Bai ( $\square$ chunling1980_0@163.com )

State Key Laboratory of Reproductive Regulation \& Breeding of Grassland Livestock, Inner Mongolia University https://orcid.org/0000-0003-0531-3973

\section{Research}

Keywords: MSTN mutant, bovine muscle satellite cells, proliferation, CDKN1C, SMAD2/SMAD3

Posted Date: June 16th, 2021

DOI: https://doi.org/10.21203/rs.3.rs-620883/v1

License: (c) (1) This work is licensed under a Creative Commons Attribution 4.0 International License. Read Full License 


\section{Abstract}

Background: Myostatin (MSTM), also known as growth/differentiation factor 8, mostly expressed in skeletal muscle and plays negative roles in regulation of muscle development. Previous studies had proved that MSTNhave important effect on cell proliferation. Therefore we aimed to investigate the mechanism of MSTNin regulating the proliferation of bovine muscle satellite cells (MSCs).

Methods: Bovine MSCs of MSTN mutant (MT) and wild type (WT) were obtained, we detected the cell proliferation and cell cycle by EdU proliferation assay and Flow cytometry. Then we detected the expression of genes associated with cell cycle by Real-time PCR and Western blotting. RNA-seq and Chromatin immunoprecipitation (ChIP)assay were performed to research the mechanism of MSTNin regulating the cell proliferation.

Results: In this study, we found that MSTN mutant promoted the proliferation of MSCs. The expression of CyclinA, CyclinD and CyclinE were all increased after MSTN mutant, while the expression of CDKN1C (P57), CDKN2A, CDKN2C and CDKN2D were down-regulated, which were consistent with the promotion of cell proliferation. Among these genes, $C D K N 1 C(P 57)$ down-regulated most significantly. RNA-seq results showed that MSTN mutant affected the SMAD binding, so we performed ChIP-qPCR and demonstrated that the SMAD2/SMAD3 transcription factor combined with the promoter of CDKN1C thus to increase the expression of $C D K N 1 C$, this demonstrating that MSTN regulated the expression of $C D K N 1 C$ through SMAD2/SMAD3 complex. Finally, overexpression of SMAD3 in wild type cells increased the expression of $C D K N 1 C$, further suggested that SMAD3 regulated the expression of CDKN1C.

Conclusion: MSTN mutant down-regulated the expression of SMAD2/SMAD3, then reduced the promotion of SMAD2/SMAD3 to the expression of CDKN1C, thus to inhibit the expression of CDKN1C, then promoting the cell cycle.

\section{Background}

Myostatin (MSTM), is a member of the transforming growth factor $\beta$ (TGF- $\beta$ ) family, also known as growth/differentiation factor-8; it is expressed in many tissues but mostly expressed in skeletal muscle, played important roles in the regulation of muscle development[1]. Many studies had proved that MSTN mutant leads to muscle hypertrophy due to both increased myofiber numbers and increased myofiber sizes, and muscle mass is the most important trait in livestock production, so MSTN has received a lot of attention. Up to now, many species of MSTN deficiency animals have been obtained, including cattle[2], humans[3], sheep[4], dogs[5], pigs[6],goats[7] and birds[8] without causing severe adverse consequences.

Previous studies had proved that MSTN have important effect on cell proliferation. Transcriptome analysis of muscle tissue of MSTN knockout chickens found that differentially expressed genes were enriched in the process of cell proliferation and differentiation[9].Knockout of MSTN promote cell proliferation, while overexpression of MSTN inhibit cell proliferation and DNA synthesis[10,11]. McCroskery et al. (2003) showed that overexpression of MSTN up-regulates P21 and downregulates 
CDK2 expression[12], MSTN negatively regulated the progression of $\mathrm{G} 1 / \mathrm{S}$ phase, thus to inhibit the cell cycle and maintain the resting state of satellite cells. Inhibition of MSTN expression by RNAi in C2C12 cells promoted the cell cycle, cells of G0/G1 phase decreased, while S phase cells increased[13,14]. Inhibition and knockout of MSTN expression by RNAi and zinc finger synthase in goat and sheep myoblasts promoted cell proliferation, decreased the expression of $P 21$ while increased $C D K 2$ expression[14,15]. But the further mechanism of MSTN regulating cell cycles were not well known. In this study, we used MSTN mutant bovine muscle satellite cells (MSCs) described in the previous study[16], detected the effect of MSTN mutant to cell cycles and the regulating pathway in the MSTN mutant bovine muscle satellite cells we obtained.

\section{Methods}

\section{Cell culture}

Muscle satellite cells (MSCs) cultured from cattle of MSTN mutant (MT) and wild type (WT) were obtained and identified as our previously reported [16]. MSCs of both MT and WT were cultured in DMEM supplemented with $10 \%$ horse serum (HS) and $20 \%$ fetal bovine serum (FBS) with $5 \% \mathrm{CO}_{2}$ at $38.5^{\circ} \mathrm{C}$. When the confluence of cells reached $100 \%$, subculture was performed.

\section{Ethynyl-2'-deoxyuridine (EdU) assays}

The proliferation of MT and WT MSCs were detected by using EdU proliferation kit(RuiBo, Guangzhou, China), the operation method is carried out according to the manufacturer's protocol. Both MT and WT bovine muscle satellite cells were incubated with EdU for $12 \mathrm{~h}$ and washed twice with PBS for 5 min each time; then fixed the cells by $4 \%$ paraformaldehyde for $30 \mathrm{~min}$, permeabilized the cells with $0.5 \%$ Triton X-

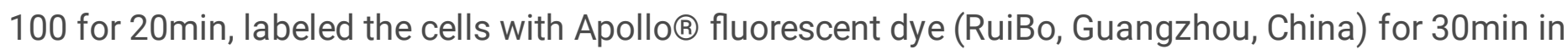
dark. Finally infiltrated the cells with PBS. Images were captured using a confocal microscope (Nikon, Tokyo, Japan) and calculated the percentage of EdU-positive cells.

\section{Myogenic cells differentiation}

Myogenic differentiation was induced when the confluence of the cultured cells reached $80-90 \%$ by using low serum medium of which $2 \%$ horse serum was supplemented to DMEM. Myogenic differentiation induction lasts for $3 \mathrm{~d}$.

\section{Flow cytometry assay}

Cell cycles were detected by the Cell Cycle and Apoptosis Analysis Kit (Beyotime, China).MSCs and differentiated MSCs were collected and washed with PBS. Next, cells were fixed for over $24 \mathrm{~h}$ in $4^{\circ} \mathrm{C}$ with $70 \%$ ethanol, then the fixed cells were washed with pre-cooled PBS and then labeled with PI solutions avoiding light for $30 \mathrm{~min}$ in $37^{\circ} \mathrm{C}$ solid bath, then the cells were detected using a flow cytometer(BD Biosciences). Cell cycles were determined by FlowJo 7.6 software. 


\section{Real-time PCR}

Expression of genes associated with cell cycle in mRNA level was detected by Real-time PCR. RNA of the myogenic cells and myotubes of MT and WT cattle were extracted by RNAiso Plus kit (9108; Takara), then the RNA was reverse-transcribed into CDNA by reverse transcription reagent (RR047A; Takara). The genes associated with cell cycle were amplified using ABI7500 real-time PCR (Applied Biosystems, America). The primers used for PCR are listed in Table S1. DNA amplification was performed as the previously described program: $95^{\circ} \mathrm{C}$ for $30 \mathrm{~s}$, followed by 40 cycles at $95^{\circ} \mathrm{C}$ for $5 \mathrm{~s}, 60^{\circ} \mathrm{C}$ for $34 \mathrm{~s}$, and a final melting curve stage [16]. The cycle threshold (Ct) values of housekeeping gene GAPDH were used to normalized the targeted genes using the $2^{-\Delta \Delta \mathrm{Ct}}$ method [17].

\section{Western blotting}

Western blotting was performed according to reference of our previously reported[16]. Briefly, the protein of cell samples were extracted using Radio Immunoprecipitation Assay (RIPA) buffer, then boiled and mixed with the loading buffer in proportion, electrophoresed in a 10\% SDS-polyacrylamide gel and transferred onto a polyvinylidene difluoride membrane by electroblotting. Then blocked the membrane, incubated with anti-CyclinA (sc-271682; Santa Cruz, USA), anti-CyclinD (sc-376676; Santa Cruz, USA), anti- P21 (sc-6246; Santa Cruz, USA), anti-P57 (sc-56341; Santa Cruz, USA) and anti-SMAD3 (ab40854; Abcam, USA) monoclonal antibodies at $1: 500$ in $4^{\circ} \mathrm{C}$ for overnight. Then washed and incubated for $1 \mathrm{~h}$ with secondary antibodies, finally captured the images using Tanon Gel Imaging System (Tanon 4800).

\section{RNA-seq analysis}

Total RNA of wild type (WT) and MSTN mutant (MT) bovine muscle tissues were extracted using TRIzol ${ }^{\circledR}$ reagent (Invitrogen). RNA sequencing was operated in Sangon Biotech company. RNA Sequencing data are shown in Table S3. Clean reads were aligned to Bos Taurus genome (NCBI:ID82) as described in reference [18]. KEGG pathway analysis and GO functional enrichment were conducted by Omicshare tools and David online database.

\section{Chromatin immunoprecipitation (ChIP) assay}

ChIP assay was performed using Pierce ${ }^{\mathrm{TM}}$ Magnetic ChIP Kit (26157; Thermo Fisher Scientific) as previously described [19]. anti-SMAD2+SMAD3 (ab202445; Abcam, USA) monoclonal antibodies was used to settle the bound DNA, the settled DNA was analyzed by quantitative PCR. Primers used for ChIPqPCR were provided in Table S2.

\section{Construction and transfection of SMAD3 overexpression plasmid}

cDNA of SMAD3 was cloned from cattle muscle tissue. The primers were as follows: $5^{\prime}-$ ATGTCGTCCATCCTGCCTTTC-3' (forward) and 5'-CTAAGACACGCTGGAGCAG-3' (reverse). The obtained SMAD3 CDNA and the PCAG-IRES2-AcGFP vector were digested by restriction endonucleases of ECoRI and BamH. Nextly, the SMAD3 fragment and the linearized pCAG-IRES2-EGFP vector were ligated. Then the 
SMAD3 overexpression vector was transfected using Lipofectamine LTX Reagent (Invitrogen Life Technologies, Carlsbad, CA, USA). Transfection was conducted when the MSCs reaching $85 \%$ confluence. RNA was isolated $48 \mathrm{~h}$ after transfection.

\section{Statistical analysis}

T-tests were used to analysis the significance difference between MSTN mutant and wild type MSCs. pvalues of less than 0.05 were considered statistically significant.

\section{Results}

\section{MSTN mutant promoted the proliferation of muscle satellite cells (MSCs)}

The muscle satellite cells (MSCs) derived from both MT and WT cattle were shown in Fig 1A, and identified in our previously reported reference[16]. We analyzed the proliferation of the MSCs isolated from MT and WT to detect the effect of MSTN mutant to cell proliferation. EdU proliferation assay results showed that MSTN mutant promoted the proliferation of MSCs(Figure 1B, 1C). Then we analyzed the cell cycle of these two cells by Flow cytometry. Results showed that cell numbers of G0/G1 and G2/M phase decreased while the cell numbers of S phase increased compared with WT(Figure 1D, 1E). This results indicated that MSTN mutant promoted the cell cycle mainly by promoting the DNA synthesis of cells.

\section{MSTN mutant promoted the myogenic diffefentiation by exiting the cell cycle earlier}

We then induced the MSCs of MT and WT to myogenic differentiation and the cell morphology was observed. Results indicated that myotubes were formed in MT cells after 1 day of myogenic differentiation, while this occurred after 2 days of myogenic differentiation. After 3 day' myogenic differentiation, there were more myotubes formed in MT cells than that in WT cells (Figure 2A). Then we analyzed the cell cycle of MSCs after 1, 2 and 3 day's myogenic differentiation in MT and WT cells. As shown in Figure 2B,2C and 2D, for MT MSCs, Cell number of G1 phase increased immediately after differentiated, while $S$ phase decreased immediately after 1 day of myogenesis. For WT MSCs, Cell number of G1 phase increased significantly after 2 days of myogenesis, while the $S$ phase increased on day 1 , but decreased after 1 day of myogenesis. These results showed that MT MSCs exited the cell cycle and began to differentiate immediately when induced to myogenic differentiation, but WT MSCs occurred these after 2 days of myogenesis induction. The cell cycles results were consistent with the cell morphology change results observed by microscope.

\section{MSTN mutant promoted cell proliferation mainly by down-regulating CDKN1C}

We then detected the expression of cell cycle related genes. Among the Cyclins we detected, the expression of CyclinA, CyclinD and CyclinE were all increased after MSTN mutant, and the most significantly increased is CyclinA (Figure 3A, 3C and 3D). The expression of CDK1, CDK2 and CDK6 were all increased(Fig $3 A)$. The expression of Cyclin dependent kinase inhibitors were not the same after MSTN mutant, $C D K N 1 C$ (P57), CDKN2A, CDKN2C and CDKN2D were down-regulated, which were consistent with 
the promotion of cell proliferation. Among these genes, $C D K N 1 C(P 57)$ down-regulated most significantly (Figure 3B, 3C, 3D). These results indicated that MSTN mutant promoted MSCs proliferation mainly by down-regulating $C D K N 1 C$, then promoting the CyclinA-CDK2 expression, thus to promote the synthesis of DNA and cell cycle.

\section{RNA-seq analysis}

To further analysis the mechanism of MSTN mutant to muscle cell proliferation and cell differentiation, we compared transcripts of MSTN mutant (MT) bovine and the controls. Skeletal muscle of six cattle were performed RNA-seq analysis consist of 3 MT cattle and 3 WT controls. DEGs were identified by Pvalue $<0.05$ at the same time |fold change (FC) $\mid>1.5$. Notably, expressions of 721 genes were found significantly changed has occurred in the MT cattle. Of these, the expressions of 74 genes were upregulated, while 647 genes were down-regulated in the MT cattle (Figure 4A). We then performed Realtime quantitative PCR to verify the expression of the most significant DEGs (Figure 4B), results were consistent with the RNA-seq results (Figure $4 \mathrm{C}$ ), proving that the transcriptome data is usable.

Succeeding Gene Ontology (GO) analysis indicated that the significantly differently expressed genes were with regard to the biological processes of muscle cell differentiation regulation, muscle cell proliferation, especially G1/S transition, and TGF- $\beta$ pathway (Figure 4D). At the same time, the DEGs were associated with SMAD binding (Figure 4D), this indicated that MSTN mutant may affect the binding of SMAD with downstream genes, thus to regulate cell proliferation.

\section{MSTN regulated the expression of CDKN1C by SMAD2/SMAD3 pathway}

Since SMAD2/SMAD3 complex were downstream transcription factors of the MSTN type II receptors, MSTN mutant increased the expression of $C D K N 1 C$, so we speculated that SMAD2/SMAD3 complex may combine with $C D K N 1 C$ promoter and regulate the expression of $C D K N 1 C$. This was proved by the transcription factor binding prediction analysis using the JASPAR database, results showed that SMAD2/SMAD3 complex bound with the promoter region of CDKN1C (NC_037355.1) from -804 to -792 (Figure 5B). Furthermore, we also confirmed the binding of SMAD2/SMAD3 complex with promoter of CDKN1C by performing a ChIP-qPCR assessment. We used an anti-SMAD2/SMAD3 monoclonal antibody to settle the DNA bound to SMAD2/SMAD3 complex, then amplified the CDKN1C promoter region from the deposited DNA. Region detected was from -926 to -757 on the CDKN1C promoter (Figure 5C).

\section{Overexpression of SMAD3 promoted the expression of CDKN1C}

To further discuss the influence of $S M A D 3$ to $C D K N 1 C$ expression, we then constructed the overexpression vector of SMAD3 (Figure6A, 6B), then transfected the WT MSCs with this overexpression vector. Results indicated that the expression of SMAD3 in the transgenic cells increased both in mRNA and protein levels as displayed in Figure 6C, 6D, and 6E. Then we detected the expression of CDKN1C, results showed that the expression of CDKN1C both in mRNA and protein levels increased significantly after overexpression of SMAD3(Figure 6F, 6G, 6H). These results indicated that SMAD3 promoted the expression of CDKN1C. So MSTN mutant decreased the expression of SMAD3, then 
suppressed the expression of CDKN1C, thus to promote the CyclinA-CDK2 complex expression, promoted DNA synthesis and cell proliferation.

\section{Discussion}

Myostatin (MSTM), is mainly expressed in skeletal muscle, and has a negative regulatory effect on the development of muscle mass[1]. Studies in many species have shown that MSTN gene mutation can cause abnormal muscle development, including an increase in the number of muscle fibers and an increase in the cross-sectional area of muscle fibers without causing severe adverse consequences, as previously reported in cattle[2], humans[3], sheep[4], dogs[5], pigs[6],goats[7] and birds[8]. Muscle mass is an important indicator in animal production, so many effective strategies have been made to product animals with increased muscle masses by preventing the expression of MSTN[7,29-23].

Previous studies had proved that MSTN have important effect on cell proliferation. Knockout of MSTN promote cell proliferation, while overexpression of MSTN inhibit cell proliferation and DNA synthesis[10,11,14]. In the present study, we studied the proliferation of MSTN mutant (MT) and wild-type (WT) bovine muscle satellite cells.

The MSTN mutant (MT) satellite cells were found to have a accelerated cell cycle than the control cells. Then we analyzed the cell cycle of these two cells by Flow cytometry. Results showed that cell number of G0/G1 phase reduced while the cell number of $S$ phase increased compared with WT, these results indicated that MSTN mutant promoted the cell cycle mainly by promoting DNA synthesis. This was consist with the previous studies[10,12]. RNA-seq results also showed that MSTN mutant regulated the cell proliferation and cell cycle, especially G1/S transition. This further demonstrated that MSTN mutant promoted the cell cycle mainly by promoting DNA synthesis.

During the process of myogenic differentiation, myoblasts will withdraw from the cell cycle and obtain the apoptotic phenotype to fuse into myotubes. In the present study, when the MT and WT MSCs were induced to myogenic differentiation, MT cells quit from the cell cycle and began to differentiation earlier than the WT controls. This was consist with the previous study $[11,24,25]$. Then we detected the cell cycle during myogenic differentiation, results showed that MT MSCs withdraw from the cell cycle and began to differentiate immediately when induced to myogenic differentiation, but WT MSCs occurred these after two days of myogenesis induction. So the promotion of myogenic differentiation due to MSTN mutant had two reasons: on one hand, MSTN mutant promoted the expression of myogenic related genes such as $M y O D$ and MyoG[26,27], then to promote myogenic differentiation; on the other hand, MSTN mutant promoted the cells withdraw from the cell cycle and began to differentiation earlier than the control, thus to promote myogenic differentiation[24].

Cell cycle progression was regulated by a series of related genes, so we detected the expression of cell cycle related genes. The expression of Cyclins and CDKs were all up-regulated, while the Cyclin dependent kinase inhibitors, such as CDKN1C (P57), CDKN2A, CDKN2C and CDKN2D were down-regulated, which were consistent with the promotion of cell proliferation. Among which the expression of CyclinA up- 
regulated most significantly and CDKN1C down-regulated most significantly, these results indicated that MSTN mutant promoted MSCs proliferation mainly by down-regulating $C D K N 1 C$, then promoting the CyclinA-CDK2 expression, thus to promote the synthesis of DNA and cell cycle.

SMAD were key transcription factors in TGF- $\beta$ pathway. In this study, we obtained DEGs from MT and WT cattle muscle tissue focused on SMAD binding, this indicated that MSTN may regulate cell cycle by affecting the binding of SMAD transcription factors with the downstream genes. At the same time, previous studies have shown that up-regulation of TGF- $\beta$ will increase the expression of CDK inhibitor p15, p16, p21 and p27 by increasing the binding of pSMAD3 to the their promoters[28]. So we intend to investigate that if $C D K N 1 C(P 57)$ can also bind with SMAD2/SMAD3. We predicted the binding of SMAD2/SMAD3 complex to the region of $C D K N 1 C$ promoter by JASPAR database, subsequently a ChIP assay were performed using an anti-SMAD2/SMAD3 monoclonal antibody. Results of ChIP-qPCR confirmed the directly bound of SMAD2/SMAD3 complex with the CDKN1C promoter region. Moreover, overexpression of SMAD3 promoted the expression of CDKN1C. Since MSTN mutations have been found to reduce the activity of SMAD2/SMAD3 complex[29,30], so in the present study, MSTN mutant decreased the promotion of SMAD2/SMAD3 to the expression of CDKN1C, so the expression of CDKN1C was decreased, and then promoted the CyclinA-CDK2 expression, thus to promote the synthesis of DNA and cell cycle.

\section{Conclusion}

In summary, the MSTN mutation leads to decreased activity of SMAD2/SMAD3, which bound directly to the promoter region of $C D K N 1 C$, SMAD3 overexpression promoted the expression of CDKN1C, so MSTN mutant down-regulated the expression of $C D K N 1 C$ by SMAD2/SMAD3 pathway. Down-regulation of CDKN1C then promoted the CyclinA-CDK2 complex, then promoted the cell proliferation.

\section{Abbreviations}

MSTN: Myostatin; MSCs:smuscle satellite cells; MT: MSTN mutant; WT: wild type; ChIP: Chromatin immunoprecipitation; KEGG: Kyoto Encyclopedia of Genes and Genomes; GO: Gene Ontology; DEGs: Differentially Expressed Genes.

\section{Declarations}

\section{Acknowledgments}

Not applicable.

\section{Authors' contributions}

The authors' contributions are as follows: Chunling Bai and Li Gao conceived and designed the study; Li Gao, Mingjuan Gu , Yunpeng Liu and Caihong Bu conducted the research, Miaomiao Yang and Peng 
Cheng analyzed and interpreted the data; L. G. wrote the manuscript. All authors read and approved the final version of the manuscript.

\section{Funding}

This work was supported by the National Science and Technology Major Project of China [grant numbers 2016ZX08007-002]; the Baotou City Youth Innovative Talents Project [grant numbers 2010,6333]; and the High-level Talents Introduced Scientific Research Startup Fund Project of Baotou Teacher's College [grant numbers BTTCRCQD2020-003].

\section{Declarations}

\section{Ethics statement}

All animal operating procedures were authorized by the Inner Mongolia University and Baotou Teacher's College Committee on the Ethics of Animal Experiments. All methods were performed compliance with the relevant policies and guidelines.

\section{Competing interests}

The authors declare that there is no conflict of interest.

\section{References}

[1]McPherron AC, Lee SJ. Double muscling in cattle due to mutations in the myostatin gene.PNAS.1997;94:12457-12461. doi:10.1073/pnas.94.23.12457

[2]Kambadur R, Sharma M, Smith TP, Bass JJ. Mutations in myostatin (GDF8) in double-muscled Belgian Blue and Piedmontese cattle. Genome Res.1997;7:910-916. doi:10.1101/gr.7.9.910

[3]Schuelke M, Wagner KR, Stolz LE, Hübner C, Riebel T, Kömen W, et al. Myostatin mutation associated with gross muscle hypertrophy in a child. N Engl J Med. 2004;350:2682-2688.

doi:10.1056/NEJMoa040933

[4] Clop A, Marcq F, Takeda H, Pirottin D, Tordoir X, Bibé B, et al. A mutation creating a potential illegitimate microRNA target site in the myostatin gene affects muscularity in sheep. Nat Genet. 2006; 38:813-818. doi:10.1038/ng1810

[5]Mosher DS, Quignon P, Bustamante CD, Sutter NB, Mellersh CS, Parker HG, et al. A mutation in the myostatin gene increases muscle mass and enhances racing performance in heterozygote dogs. PLoS Genet. 2007;3:e79. doi:10.1371/journal.pgen.0030079

[6] Bi Y, Hua Z, Liu X, Hua W, Ren H, Xiao H, et al. Isozygous and selectable marker-free MSTN knockout cloned pigs generated by the combined use of CRISPR/Cas9 and Cre/LoxP. Sci Rep. 2016; 
[7] Wang X, Niu Y, Zhou J, Zhu H, Ma B, Yu H, et al. CRISPR/Cas9-mediated MSTN disruption and heritable mutagenesis in goats causes increased body mass. Anim Genet.2018;49(1):43-

51. doi:10.1111/age. 12626

[8]Lee J, Kim DH, Lee K. Muscle Hyperplasia in Japanese Quail by Single Amino Acid Deletion in MSTN Propeptide. Int J Mol Sci. 2020;21(4);1504. doi:10.3390/ijms21041504

[9] Xu K, Han CX, Zhou H, Ding JM, Xu Z, Yang LY, et al. Effective MSTNGene Knockout by AdV-Delivered CRISPR/Cas9 in Postnatal Chick Leg Muscle. Int J Mol Sci. 2020; 21(7):2584. doi:10.3390/ijms21072584

[10]Taylor WE, Bhasin S, Artaza J, Byhower F, Azam M, Willard DH, et al. Myostatin inhibits cell proliferation and protein synthesis in C2C12 muscle cells. Am J Physiol Endocrinol Metab. 2001;280(2):E221-E228. doi:10.1152/ajpendo.2001.280.2.E221

[11]Ríos R, Carneiro I, Arce VM, Devesa J. Myostatin regulates cell survival during C2C12 myogenesis. Biochem Biophys Res Commun.2001;280(2):561-566. doi:10.1006/bbrc.2000.4159

[12]McCroskery S, Thomas M, Maxwell L, Sharma M, Kambadur R. Myostatin negatively regulates satellite cell activation and self-renewal. J Cell Biol. 2003; 162(6):1135-1147. doi:10.1083/jcb.200207056

[13]Liu C, Yang Z, Zhao B, Liu C. Inhibiting GDF-8 expression by retrovirus-based RNAi stably. Sheng Wu Gong Cheng Xue Bao.2008;24(2):250-255.

[14]Salabi F, Nazari M, Chen Q, Nimal J, Tong J, Cao WG. Myostatin knockout using zinc-finger nucleases promotes proliferation of ovine primary satellite cells in vitro. J Biotechnol. 2014;192 Pt A:268280. doi:10.1016/j.jbiotec.2014.10.038

[15]Patel AK, Tripathi AK, Patel UA, Shah RK, Joshi CG.Myostatin knockdown and its effect on myogenic gene expression program in stably transfected goat myoblasts. In Vitro Cell Dev Biol Anim.2014;50(7):587-596. doi:10.1007/s11626-014-9743-4

[16]Gao L, Yang MM, Wei ZY, Gu MJ, Yang L, Bai CL,et al. MSTN Mutant Promotes Myogenic Differentiation by Increasing Demethylase TET1 Expression via the SMAD2/SMAD3 Pathway. Int J Biol Sci.2020;16(8),1324-1334. doi:10.7150/ijbs.40551

[17]Shen SJ, Zhang YH, Gu XX, Jiang SJ, Xu LJ. Yangfei Kongliu Formula, a compound Chinese herbal medicine, combined with cisplatin, inhibits growth of lung cancer cells through transforming growth factor- $\beta 1$ signaling pathway. J Integr Med. 2017;15(3):242-251. doi:10.1016/S2095-4964(17)60330-3

[18] Trapnell C, Pachter L, Salzberg SL. TopHat: discovering splice junctions with RNA-Seq. Bioinformatics.2009;25(9):1105-1111. doi:10.1093/bioinformatics/btp120 
[19]Gao L, Yang M, Wang X, Yang L, Bai C, Li G. Mstn knockdown decreases the trans-differentiation from myocytes to adipocytes by reducing Jmjd3 expression via the SMAD2/SMAD3 complex. Biosci Biotechnol Biochem.2019;83(11):2090-2096. doi:10.1080/09168451.2019.1644152

[20]Fiems LO. Double Muscling in Cattle: Genes, Husbandry, Carcasses and Meat. Animals (Basel).2012;2(3):472-506. doi:10.3390/ani2030472

[21] Yu B, Lu R, Yuan Y, Zhang T, Song S, Qi Z, et al.Efficient TALEN-mediated myostatin gene editing in goats. BMC Dev Biol.2016;16(1):26. doi: 10.1186/s12861-016-0126-9.

[22] Wang K, Tang X, Xie Z, Zou X, Li M, Yuan H, et al. CRISPR/Cas9-mediated knockout of myostatin in Chinese indigenous Erhualian pigs. Transgenic Res. 2017;26(6):799-805. doi: 10.1007/s11248-017-0044Z.

[23] Zhang J, Cui ML, Nie YW, Dai B, Li FR, Liu DJ, et al. CRISPR/Cas9-mediated specific integration of fat1 at the goat MSTN locus. FEBS J. 2018;285(15):2828-2839. doi: 10.1111/febs.14520.

[24]Joulia D, Bernardi H, Garandel V, Rabenoelina F, Vernus B, Cabello G. Mechanisms involved in the inhibition of myoblast proliferation and differentiation by myostatin. Exp Cell Res. 2003;286(2):263-275. doi:10.1016/s0014-4827(03)00074-0

[25]Zhang Y, Wang Y, Yulin B, Tang B, Wang M, Zhang C, et al. CRISPR/Cas9-mediated sheep MSTN gene knockout and promote sSMSCs differentiation. J Cell Biochem. 2018;120(2):17941806. doi:10.1002/jcb. 27474

[26]Liu CX, Li WR, Zhang XM, Zhang N, He SG, Huang JC, et al. The critical role of myostatin in differentiation of sheep myoblasts. Biochem Biophys Res Commun.2012;422(3):381386. doi:10.1016/j.bbrc.2012.04.151

[27]Du W, Zhang Y, Yang JZ, Li HB, Xia J, Li N, et al.Effect of MSTN propeptide protein on the growth and development of Altay lamb muscle. Genet Mol Res. 2016;15(2):gmr7778. doi:10.4238/gmr.15027778

[28]Carlson ME, Hsu M, Conboy IM. Imbalance between pSmad3 and Notch induces CDK inhibitors in old muscle stem cells. Nature.2008;454(7203):528-532. doi:10.1038/nature07034

[29]Langley B, Thomas M, Bishop A, Sharma M, Gilmour S, Kambadur R. Myostatin inhibits myoblast differentiation by down-regulating MyoD expression. J Biol Chem. 2002;277(51):49831-49840. doi:10.1074/jbc.M204291200

[30]Rebbapragada A, Benchabane H, Wrana JL, Celeste AJ, Attisano L.Myostatin signals through a transforming growth factor beta-like signaling pathway to block adipogenesis. Mol Cell Biol. 2003;23(20):7230-7242. doi: 10.1128/mcb.23.20.7230-7242.2003 


\section{Figures}

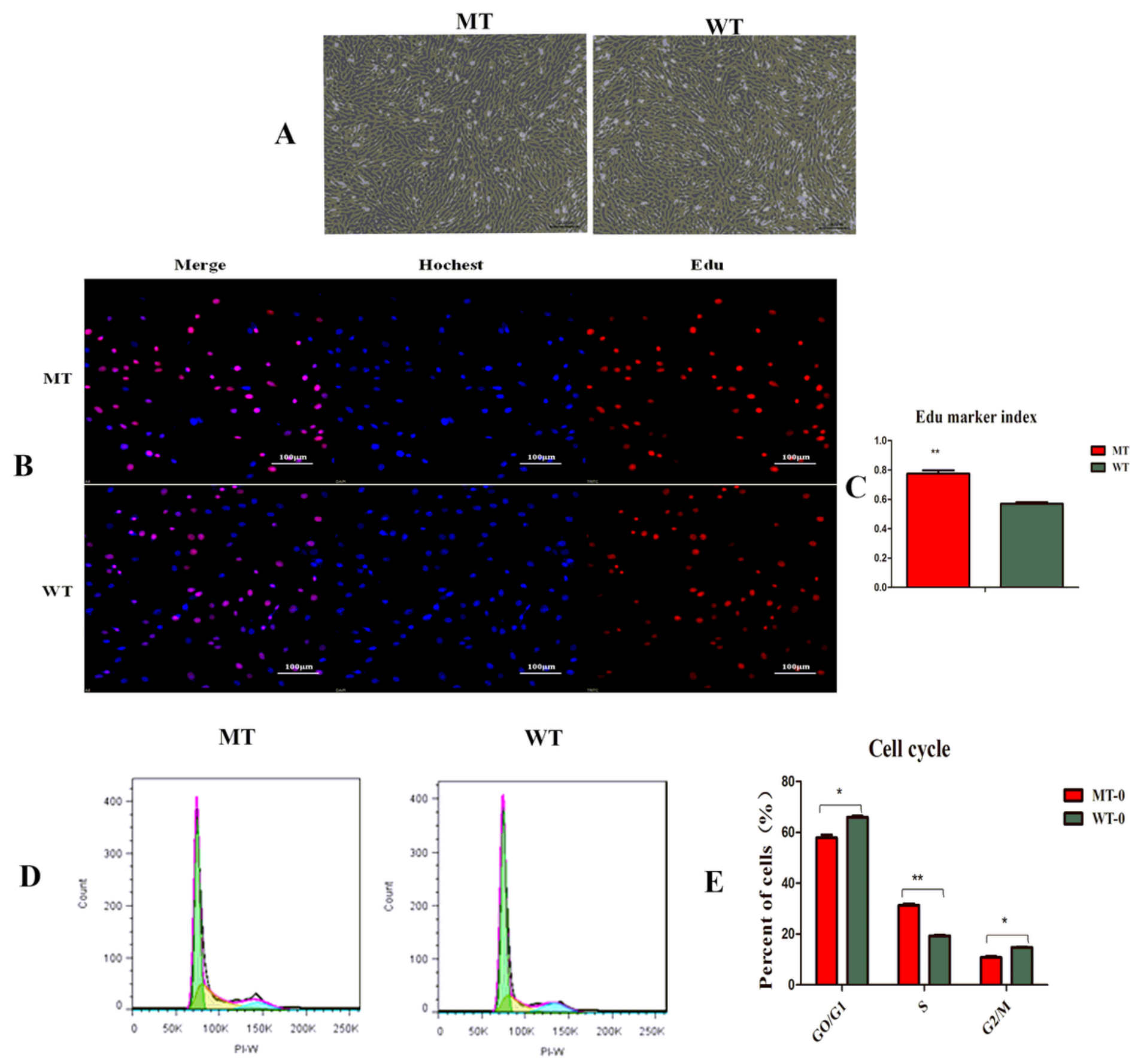

Figure 1

MSTN mutant promoted the proliferation of muscle satellite cells (MSCs). A, Morphology of the WT and MSTN mutant (MT) bovine muscle cells. Magnification of microscope is 50x. B, Proliferation of MSCs was analyzed by EdU assays. Blue, cell nuclei stained with Hoechst 33342. Red, EdU staining; C, Edu marker index (the number of new synthesized nuclei (red)/the number of all nuclei (blue), n=5).D,Cell cycle of MT and WT MSCs analyzed by Flow cytometry. E, cell Cycles $(n=3)$. T-test was used to generate p-value. ${ }^{*} p<0.05,{ }^{* \star} p<0.01,{ }^{\star \star *} p<0.001$. 
A

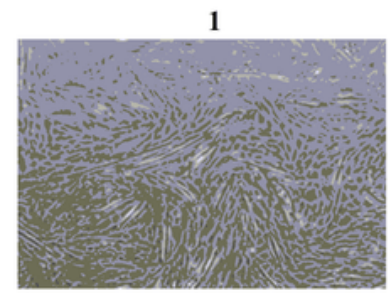

WT

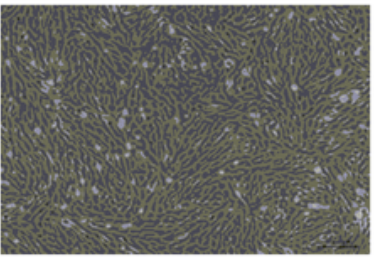

1

B
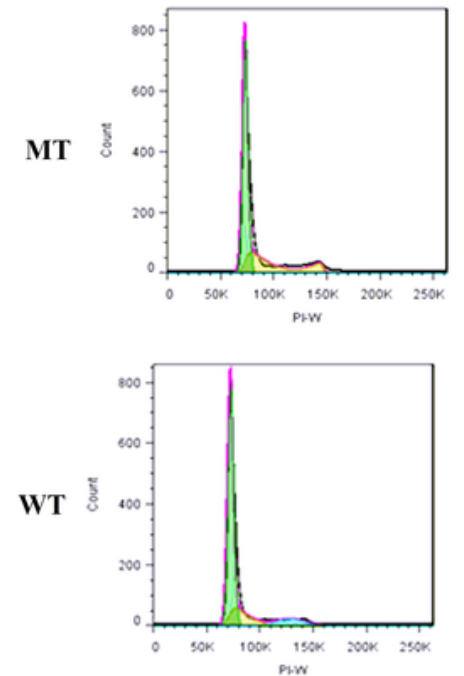

Cell cycle

C

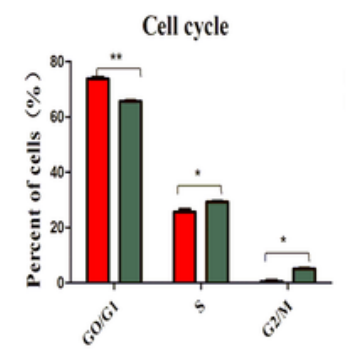

G0/G1

D

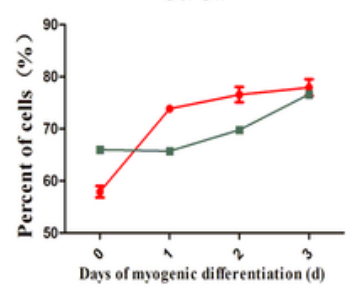

2
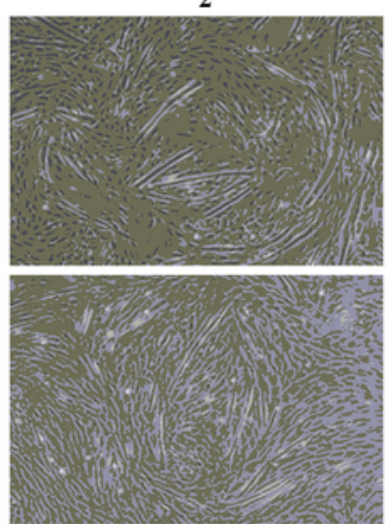

2
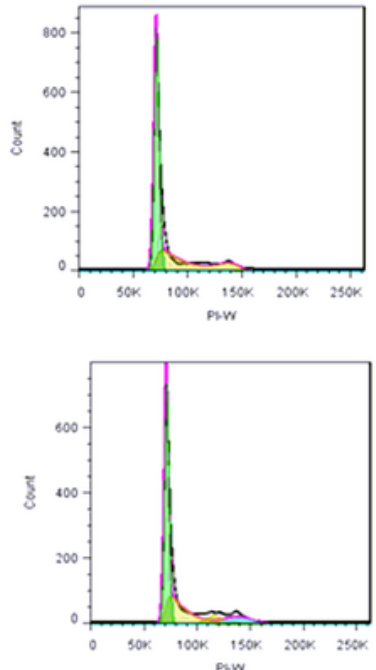

Cell cycle

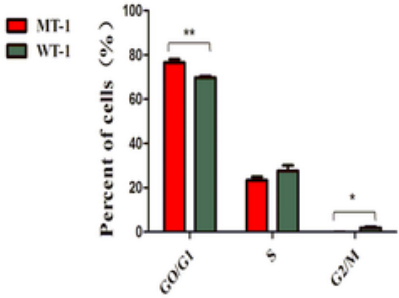

S

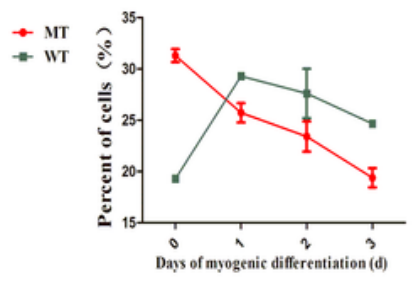

3
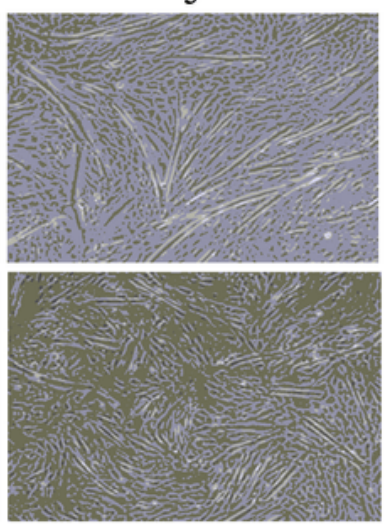

3
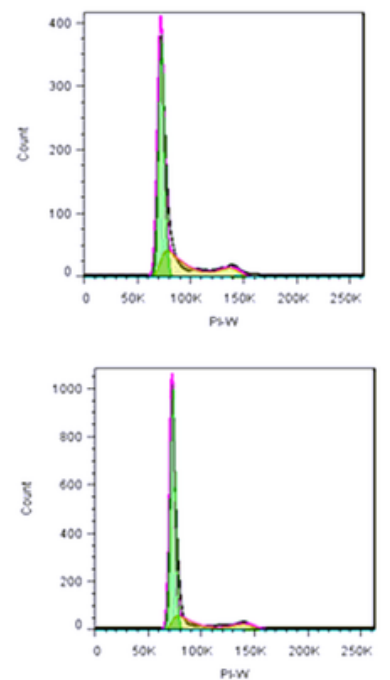

Cell cycle

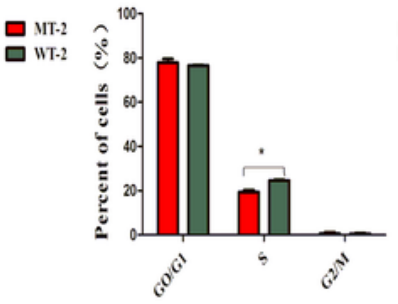

G2/M

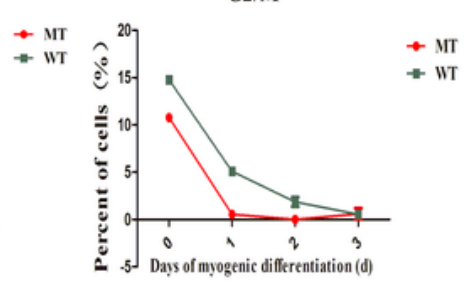

\section{Figure 2}

MSTN mutant promoted the myogenic diffefentiation by exiting the cell cycle earlier. A,The differentiated MT and WT MSCs of 1, 2 and 3 days. MT MSCs began to differentiate on day 1 when induced to myogenic differentiation, while WT MSCs began to differentiate on day 2 when induced to myogenic differentiation. B, Cell cycle of differentiated cells analyzed by Flow cytometry. C, Counted cell cycles 
$(n=3)$. D, Changes in the proportion of cells in each stage of MT and WT cells after induced to myogenesis. T-test was used to generate $p$-value. ${ }^{*} p<0.05,{ }^{* *} p<0.01,{ }^{* \star *} p<0.001$.

\section{Expression of Cyclins and CDKs}

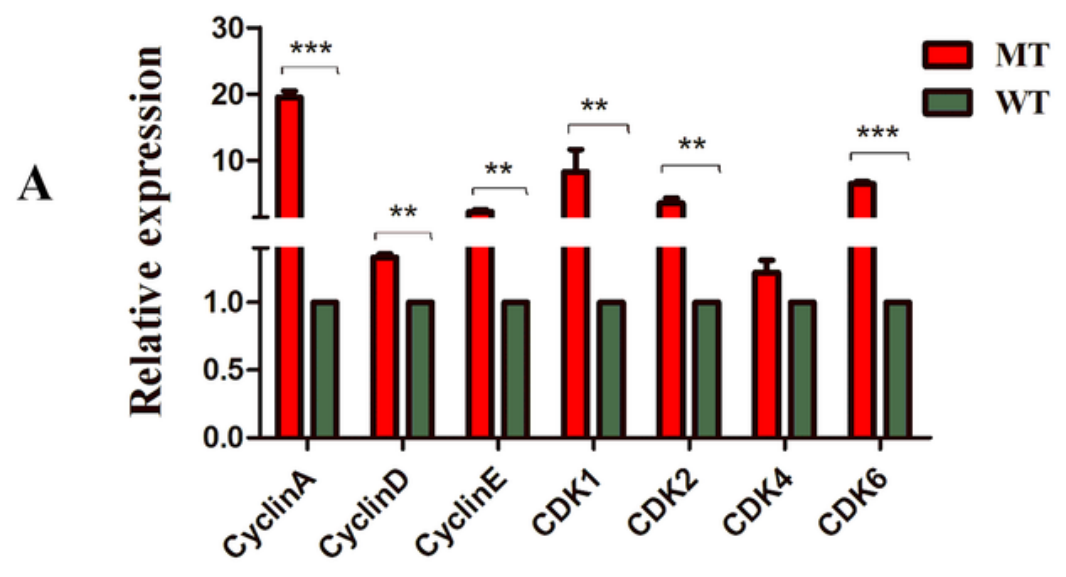

Expression of cyclin dependent kinase inhibitors
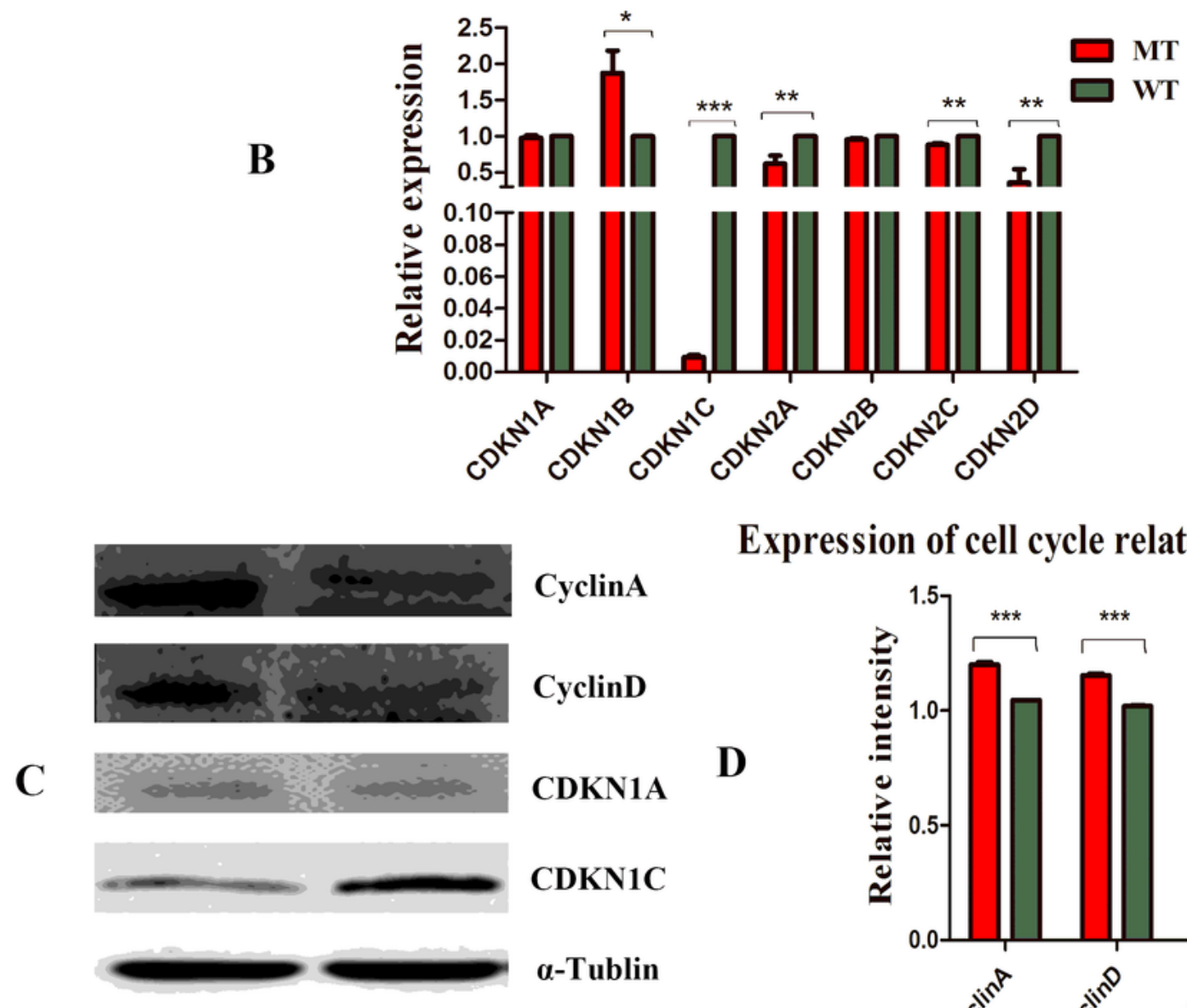

Expression of cell cycle related genes in protein level

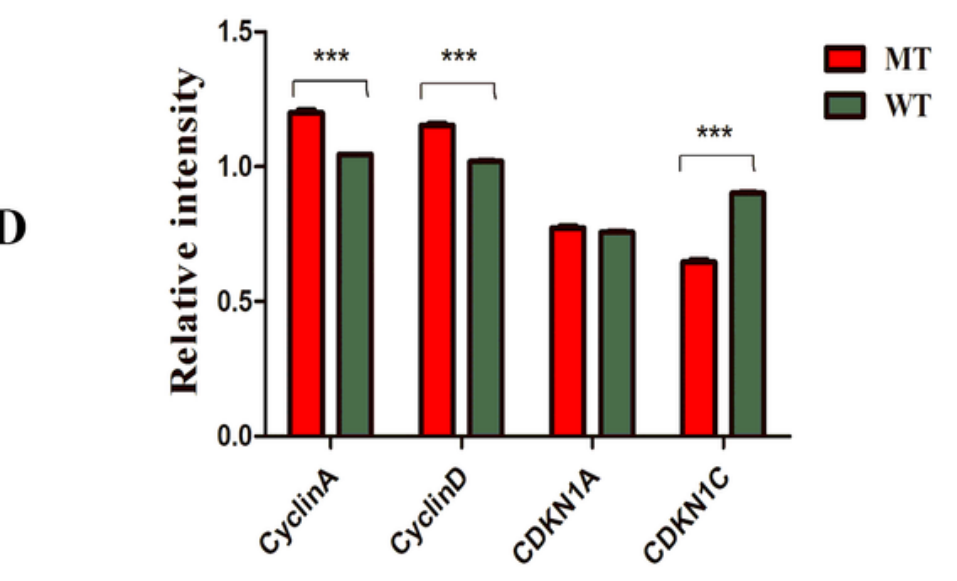

Figure 3

MSTN mutant promoted cell proliferation mainly by down-regulating CDKN1C. A, Expression of CDKs and Cyclins in mRNA level. B, Expression of cyclin dependent kinase inhibitors in mRNA level. C, Expression of 
Cyclins and cyclin dependent kinase inhibitors in protein level detected by western blot and quantified in D. T-test was used to generate $p$-value. ${ }^{\star} p<0.05,{ }^{\star \star} p<0.01,{ }^{\star \star \star} p<0.001$.

A

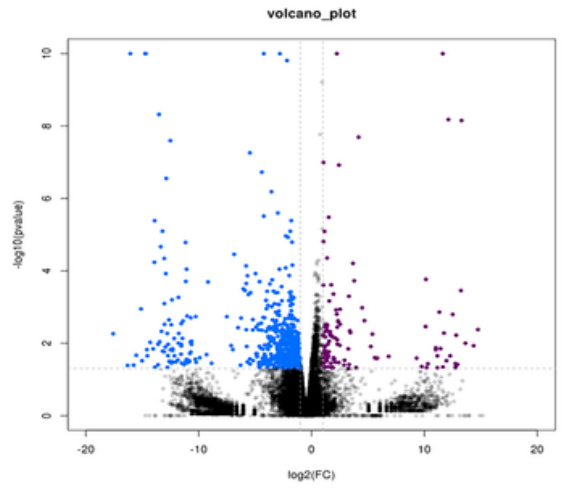

- down

- up

- nodiff
B

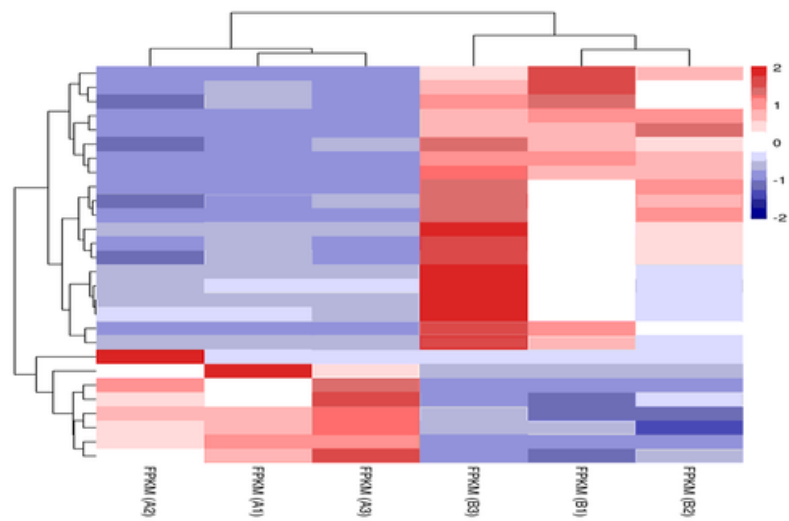

Real-time PCR for RNA-Seq results

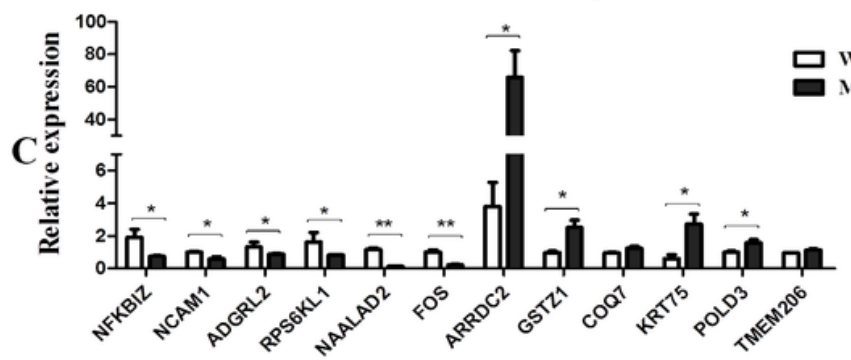

D

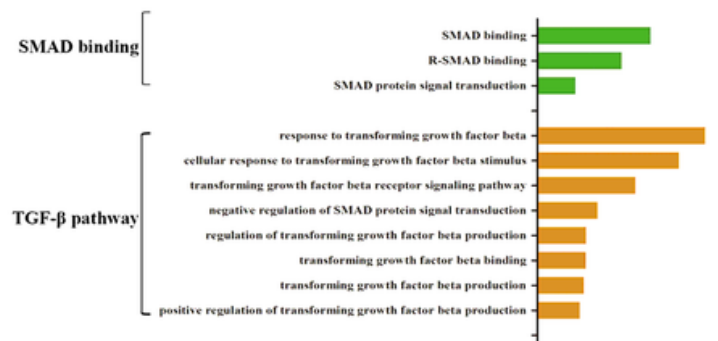

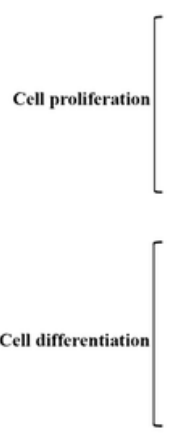

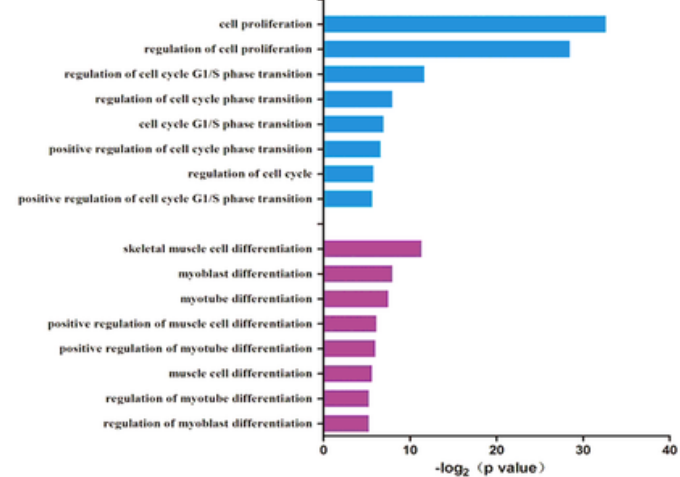

Figure 4

RNA-seq analysis. A, The differential expression genes (DEGs) between MSTN gene edited and control cattle muscle tissues. B, Heatmap of the most 30 significant DEGs between MSTN gene edited and control cattle muscle tissues. Red represents the up-regulated genes, blue represents the down-regulated genes. C, qPCR data for differential expression genes. D, GO enrichment analysis of DEGs. The DEGs were enriched on GO terms of cell differentiation, cell proliferation, TGF- $\beta$ pathway and SMAD binding. 


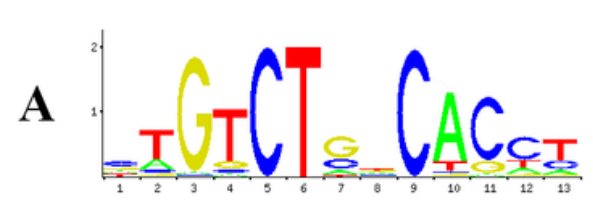

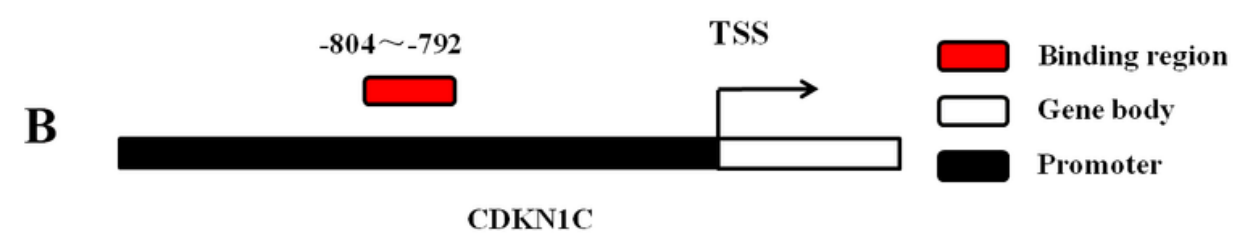

\section{Combination of SMAD2/SMAD3 with CDKN1C}

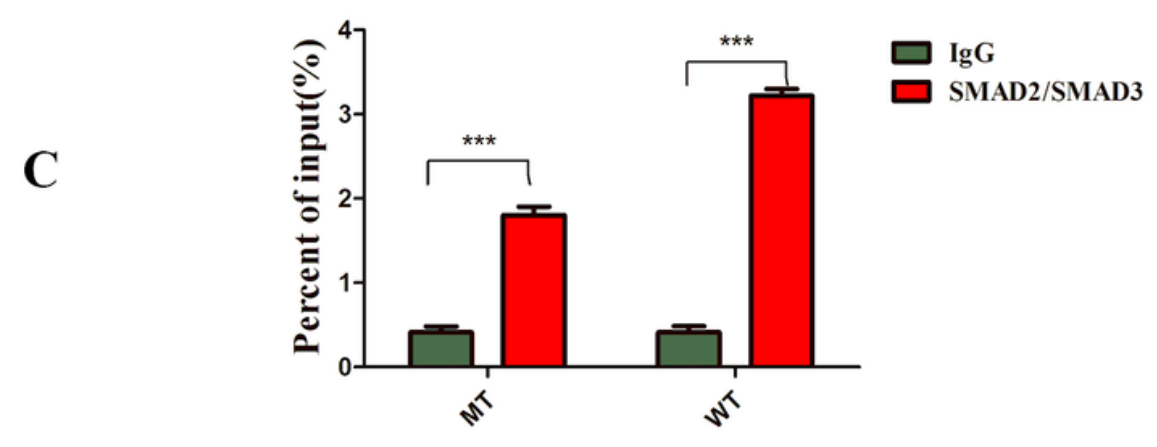

Figure 5

MSTN regulated CDKN1C expression by SMAD2/SMAD3 pathway. A, The motif of SMAD2/SMAD3 transcription factor. $B$, Binding region of SMAD2/SMAD3 complex with CDKN1C promoter. C, The binding of CDKN1C promoter with SMAD2/SMAD3 complex detected by ChIP-qPCR. The binding region on the promoter was from -804 to -792 of CKDN1C. T-test was used to generate $p$ value. ${ }^{*} p<0.05$, ${ }^{* *} p<0.01$, ${ }^{\star * *} p<0.001$. 

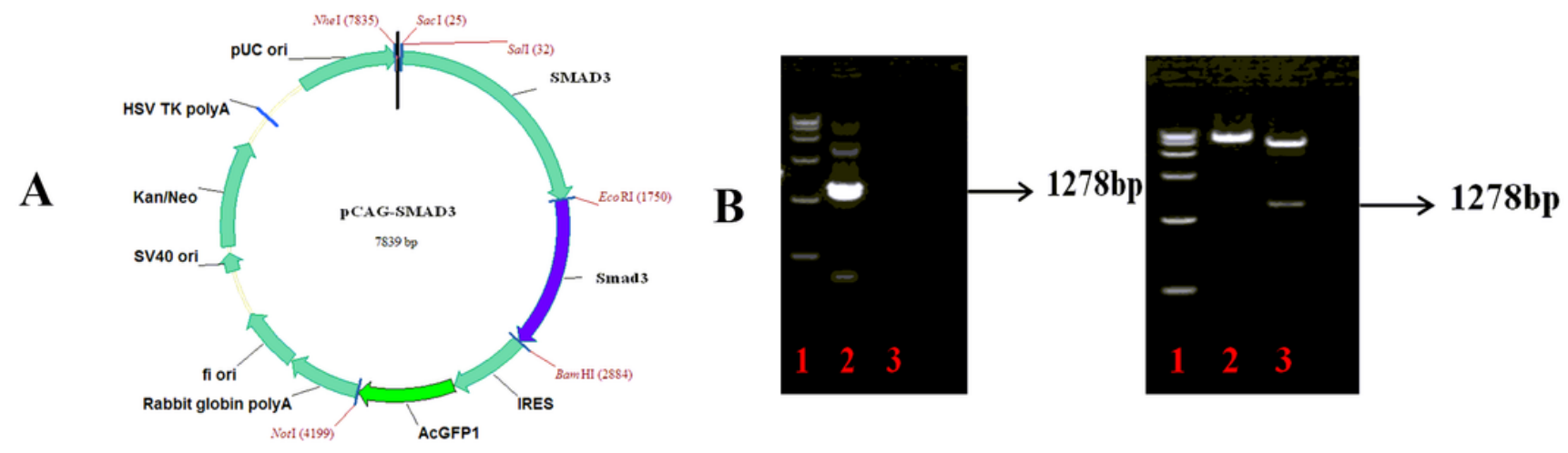

Expression of SMAD3 with SMAD3 overexpression

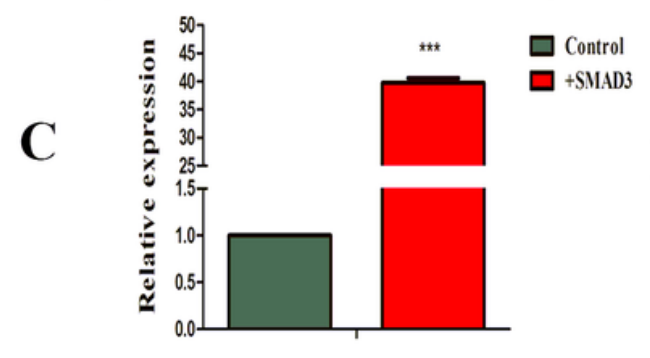

D

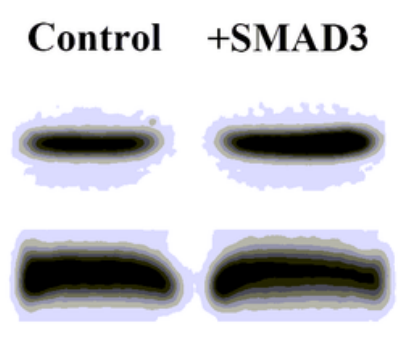

SMAD3

$\alpha$-Tublin

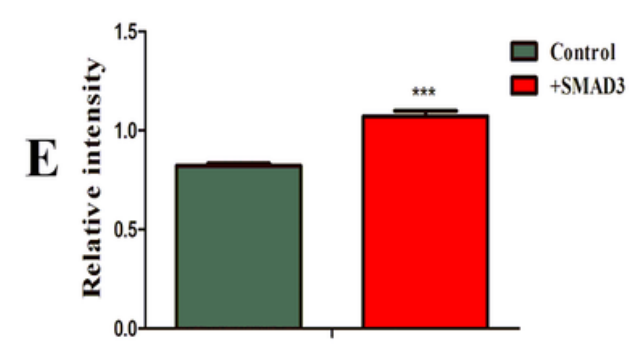

Expression of CDKN1C with SMAD3 overexpression
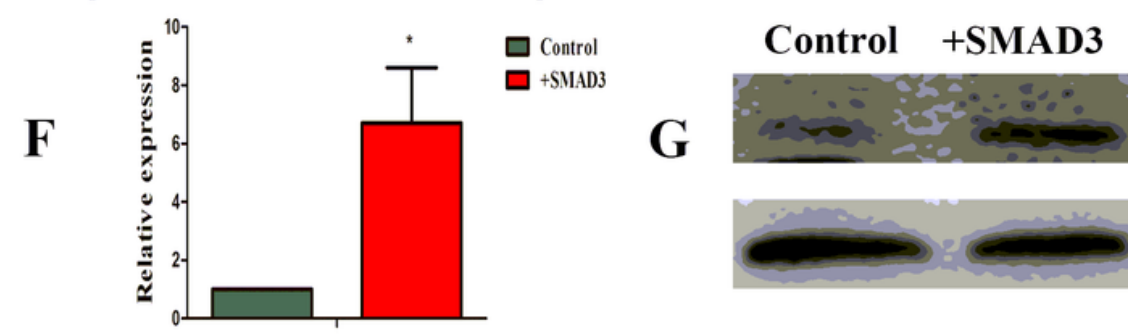

P57

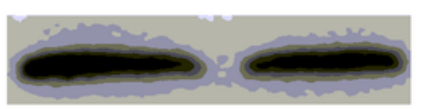

$\alpha$-Tublin

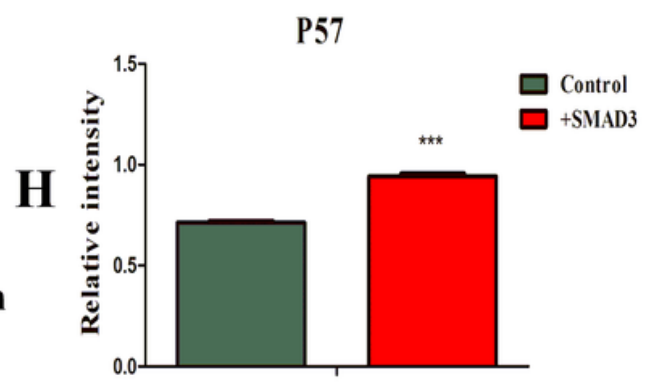

Figure 6

Overexpression of SMAD3 promoted the expression of CDKN1C. A, The overexpression vector of SMAD3. B, left: identification of the pCAG-SMAD3-IRES-AcGFP plasmid by PCR, 1,DL 5000 marker; 2, plasmid; 3, ddH2O; right: identification of the pCAG-SMAD3-IRES-AcGFP plasmid by restriction enzyme digestion. 1, DL 5000 marker; 2, Single digestion by EcoRI; 3, Double digestion by EcoRI and BamHI. C, Expression of SMAD3 in mRNA level after overexpression of SMAD3 to muscle satellite cells of wild type; $D$ and $E$, Expression of SMAD3 in protein level. F, Expression of CDKN1C in mRNA level after overexpression of SMAD3 to wild type muscle satellite cells. $G$ and $H$, Expression of CDKN1C in protein level. T-test was used to generate $p$-value. ${ }^{\star} p<0.05,{ }^{\star \star *} p<0.01,{ }^{\star \star *} p<0.001$.

\section{Supplementary Files}

This is a list of supplementary files associated with this preprint. Click to download. 
- SupplementaryFiles.pdf

Page 18/18 